\title{
Free Surface Shock Waves and Methods for Hull Form Improvement
}

\author{
(Second Report)
by Hideaki Miyata*, Member
Masao Nito**, Member
Mitsuyasu Nagahama****, Member Yoshihiro Tsuchiya*
Hisashi Kajitani*, Member
Kiyohira Aoki***, Member

\begin{abstract}
Summary
The effect of bow-bulbs on reduction of wave resistance is studied through some kinds of experiment with wedge models and a ship model of fine hull form. The stress is focused on their effects on wave configuration and flow velocities in the near-field. The effect of frame-lines of hull forms on both linear and nonlinear wave resistance components is also studied with a ship model of fine practical hull form. An approximate method of estimating the two resistance components is proposed and applied to hull form improvement.
\end{abstract}

\section{Introduction}

In the first report ${ }^{1)}$, a method of optimizing sectional area curves of ships was proposed, which utilizes the relation between hull form and resistance coefficient, and it was incorporated into the wave-analytical procedure of obtaining hull forms of minimum wave pattern resistance. The informations from the wave profiles in the near-field must be inevitably taken into consideration for the accomplishment of the empirical procedure of minimizing wave resistance. The availability of the new optimization method was demonstrated by the experiments with fine ship models of container carrier.

There are some other parameters of hull forms in addition to sectional area curve, and they should be considered to complete the optimization method. In this second report the effects of bow-bulbs and frame-lines on the reduction of linear and nonlinear components of wave resistance are studied.

In Chapter 2, the effect of bow-bulbs is experimentally investigated by photographing wave

* Department of Naval Architecture, The University of Tokyo

** Mitsui Engineering and Shipbuilding Co., Ltd.

*** Graduate School, The University of Tokyo

**** Hitachi Shipbuilding and Engineering Co., Ltd. formation and measuring velocity components in the near-field of wedge models and a simple hull form. The study is confined to experimental analyses and the study of the scheme of optimizing bow-bulbs is postponed. In Chapter 3, a method of estimating wave resistance of ships whose frame-lines are systematically modified is proposed, which is considerably different from the method proposed in the first report, although the previous method is also applicable if three ship models are served for experiments. This method is employed for the optimization of the frame-lines of forebodies.

The denotation of variables and parameters is as shown in the nomenclature of the first report, unless otherwise defined.

\section{Effect of bow-bulbs}

Most of the marchant ships have been equipped with bow-bulbs since Inui and others developed gigantic bow-bulbs and demonstrated the mechanism of wave cancellation. They showed that the reduction of wave resistance by bow-bulbs is revealed in the cancellation of linear dispersive waves generated by main hulls and bow-bulbs. In 1970's Sharma and Baba showed that the reduction of wave resistance by bow-bulbs of low-speed full hull forms does not appear in wave pattern resistance derived from wave analysis in the far-field. Some recent studies at The Experiment Tank of The University of Tokyo indicated that bow-bulbs reduce nonlinear waves 
in the near-field of ships called free surface shock wave (FSSW) as well as linear dispersive waves.

The investigation into the structure of free surface shock waves ${ }^{3)}$ gives a little more comprehensive understanding of the complicated nonlinear wave making phenomenon. The waves generated by advancing bodies in the near-field have substantially nonlinear characteristics which are analogous to other nonlinear wave phenomena. The generation of steep nonlinear waves and singular wave pattern formation are the fundamental aspects of free surface shock waves. These nonlinear configurations of waves involve breaking of crests and energy deficit at the second stage, and the dissipative property of FSSW results in momentum loss far behind. The nonlinear waves in the near-field, on the other hand, are surrounded by dispersively spreading linear waves, which implies the possibility of the wave energy of FSSW to be partly supplied to the linear dispersive waves. It is supposed that waves in the near-field is nonlinear ones called free surface shock wave, and their energy is partly dissipated and partly spread dispersively. The importance of either dissipation or dispersion depends on particulars of ships and speed of advance.

Linear wave making theories cannot cope with the substantial phenomenon of wave making in the near-field, and they also have difficulties in estimating the wave resistance due to linear dispersive waves in the far-field, because the waves in the far-field are seriously influenced by the nonlinear effects of FSSW. Therefore, the effect of bow-bulbs on wave making must be clarified by examining the behavior of nonlinear waves in the near-field.

\subsection{Wedge models and their bulbs}

The combination of a wedge and a horizontal cylinder with spherical head is chosen as a most simplified model of experiments on bow-bulbs. The wedge models used for the experiments to study the characteristics of $\mathrm{FSSW}^{2}$ are made use of for the present purpose. The length and depth of the models are about $1.0 \mathrm{~m}$ and $0.35 \mathrm{~m}$, respectively. The half-entrance angle $(\alpha)$ is $10^{\circ}$, $15^{\circ}$ and $20^{\circ}$. The bow-bulbs are of horizontal cylinder type whose diameter is $80 \mathrm{~mm}$, as shown in Fig. 1. The protrusion of the bulbs is $60 \mathrm{~mm}$ (F1-bulb) and $100 \mathrm{~mm}$ (F2-bulb). The draft of the mcdels is $0.10 \mathrm{~m}$ or $0.15 \mathrm{~m}$.

The models were towed with their trim and sinkage restricted. Wave pattern pictures were taken by a $35 \mathrm{~mm}$ camera and three stroboflush-lights. Wave height was measured by a wave recorder of contact type and velocity components of the flow were measured by a 5hole pitot tube whose diameter of the sphere

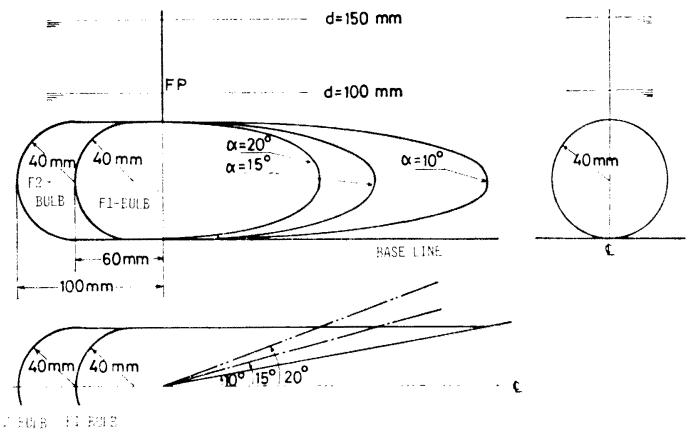

Fig. 1 Configuration of bulbs

was $6 \mathrm{~mm}$.

\subsection{Waves around wedge models with and without bulbs}

Typical wave pattern pictures are present in Figs. 2 and 3 showing the difference of wave formation due to the attachment of bulbs at $F_{d}$ (draft-base Froude number) $=1.01$. In most cases bow-bulbs reduce the angle of the wave front line to the centerline (shock angle $\beta$ ) of the foremost wave as seen in the case of $\alpha=20^{\circ}$. However, the shock angle of the foremost wave of the case of $\alpha=10^{\circ}$ is scarcely reduced, and on the contrary, that of the second wave is magnified. A gigantic bulb which is not smoothly connected to the main hull is not suitable for bows of fine entrance. The difference of protrusion does not give serious influence on the wave pattern formation in the near-field in the present cases.

Perspective views of bow wave configuration derived from measured wave height record are shown in Fig. 4 in which wave height is threetimes magnified in comparison to the horizontal length. It is obviously noted that the height of the foremost wave is considerably attenuated and simultaneously the shock angle is reduced by attaching a bulb to the wedge model of $\alpha=20^{\circ}$. A properly designed bulb reduces shock angle and it is usually accompanied by attenuation of wave height.

The variation of shock angle substantially depends on entrance angle and speed of advance and it is influenced by bulbs as shown in Figs. 5 and 6 . The variation of the shock angle of the foremost wave of the case of $\alpha=10^{3}$ is not influenced by bulbs, while the case of $\alpha=20^{3}$ shows remarkable shift of the curve of the variation of shock angle. The variation of shock angle of wedge models without bulbs is very systematic being governed by entrance angle and $F_{d}$ (sce Fig. 15 of Ref. 3)), and bulbs play the role of virtually reducing the entrance angle. 

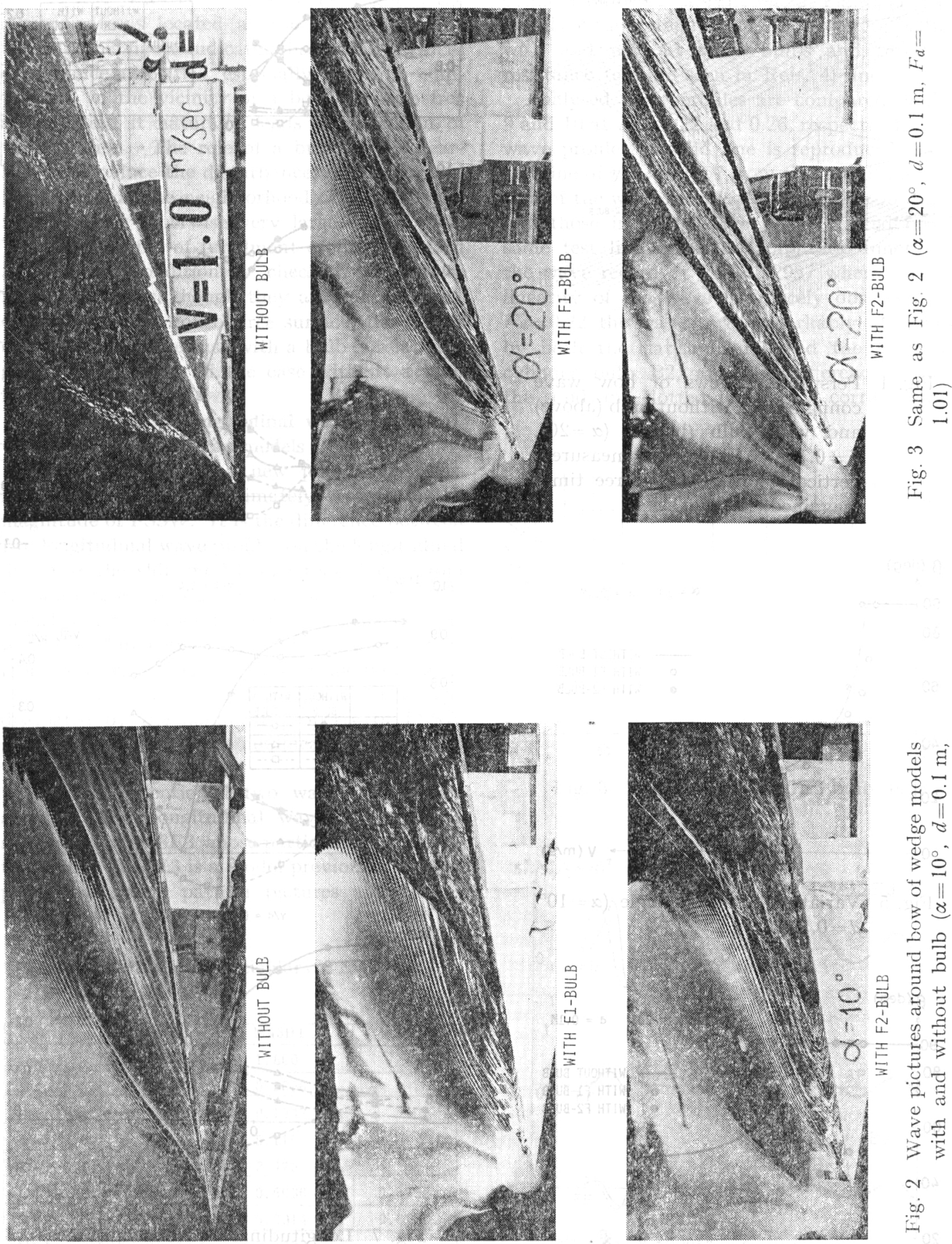

$\frac{\infty}{4}$
$\stackrel{7}{0}=$

$\exists=$

$\overbrace{0}^{\infty}$

38

○

ช

$\because \frac{0}{3}$

อี

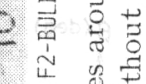

吾

듬

点焉

a

$\dot{00}$ 


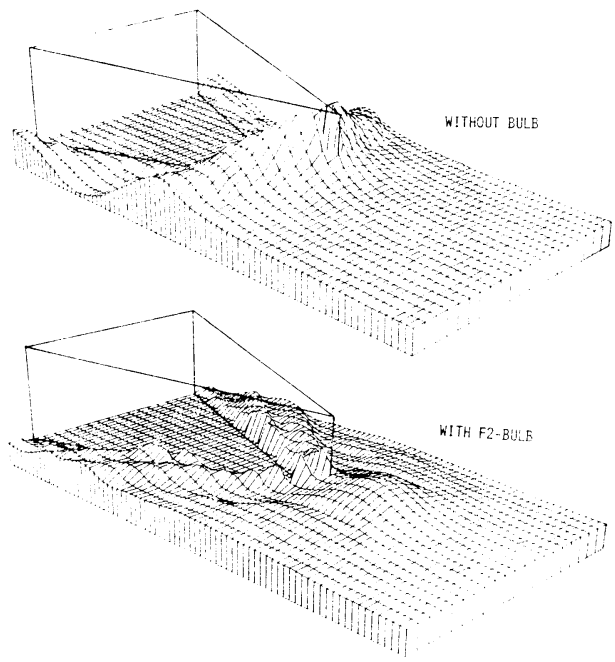

Fig. 4 Perspective views of bow wave configuration without bulb (above) and with bulb (below) $\left(\alpha=20^{\circ}\right.$, $d=0.1 \mathrm{~m}, \quad F_{d}=1.01$, measured, vertical coordinate is three times magnified)

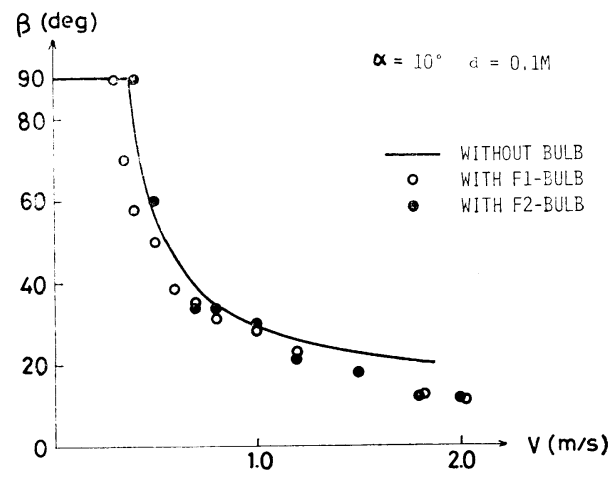

Fig. 5 Variation of shock angle $\left(\alpha=10^{\circ}\right.$, $d=0.1 \mathrm{~m})$

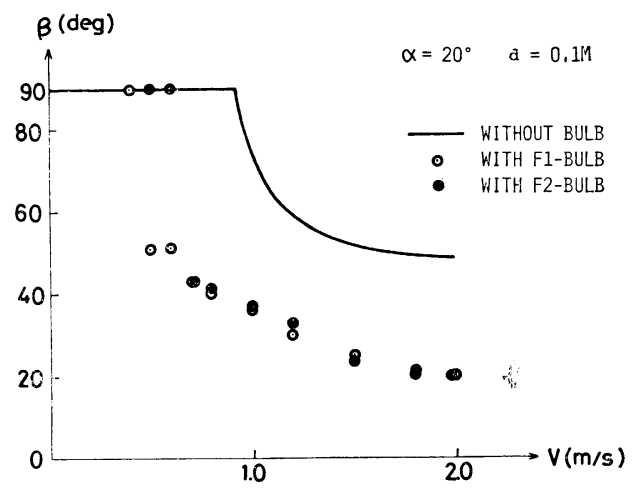

Fig. 6 Same as Fig. $5\left(\alpha=20^{\circ}, d=0.1 \mathrm{~m}\right)$
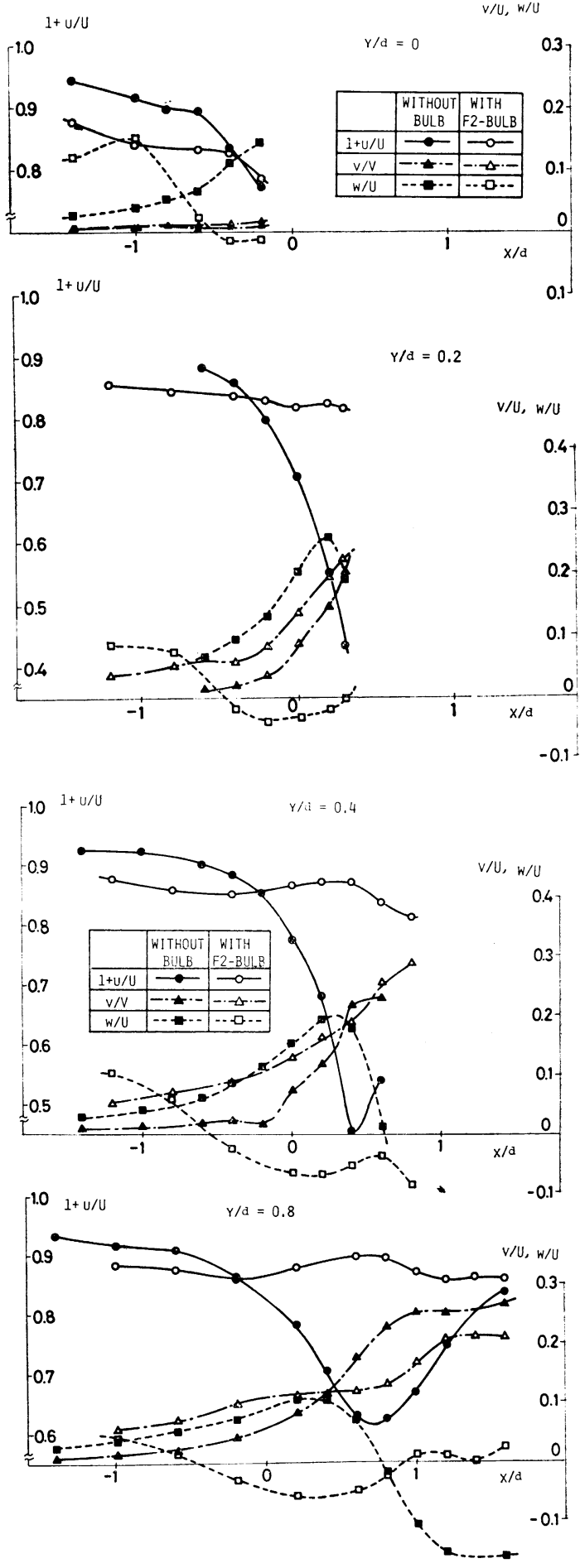

Fig. 7 Longitudinal distribution of measured velocity components on the line $15 \mathrm{~mm}$ below the disturbed free surface $\left(\alpha=20^{\circ}, \quad d=0.1 \mathrm{~m}\right.$, $F_{d}=1.01$ ) 
Velocity components measured on four longitudinal lines are compared between the two conditions, without bulb and with F2-bulb, in Fig. 7 for the case of $\alpha=20^{\circ}$, in which the origin of the $x$-coordinate is located at the fore-end of the wedge. This figure indicates that a bulb reduces disturbance velocities, especially $u$ and $w$ components, in the vicinity of a bow near the free surface, and it naturally leads to reduction of wave motion. The role of a bulb in the nearfield is to reduce the disturbance velocity caused by the bow in the neighborhood of the free surface which is otherwise very large and abrupt. When the degree of fulfillment of the linearized free surface condition is checked with these measured values, though they are not velocities on the exact location of free surface, the degree of fulfillment of the case with a bulb is relatively good, whereas that of the case without a bulb is unsatisfactory.

\subsection{Analysis of longitudinal wave profiles of M43E3 bulb series models}

In the first report, a new parameter $\Delta h$ is introduced which approximately represents the magnitude of FSSW. It is the difference between two longitudinal wave profiles on the longitudinal line near the ship model, one reproduced from the amplitude function on the line near the ship model and the other from that on the line far from the ship model. This new parameter is, more exactly, the degree of nonlinearity of waves in the near-field or the degree of wave energy dissipated by the nonlinear property of FSSW which corresponds to the nonlinear component of wave resistance.

The above-mentioned two wave profiles are analysed from longitudinal wave records of a ship model of M43E3 whose particulars are shown in Table 1. M43E3 is a model previously used to photograph wave pattern pictures and to ex-

Table 1 Principal particulars of M43 bowbulb series

\begin{tabular}{|c|c|c|c|}
\hline$(\mathrm{m})$ & $\mathrm{M} 43 \mathrm{E} 3$ & M43B1 & M43B 2 \\
\hline Lpp & \multicolumn{3}{|c|}{1.860} \\
\hline B & \multicolumn{3}{|c|}{0.3636} \\
\hline $\mathrm{d}$ & \multicolumn{3}{|c|}{0.1047} \\
\hline $\mathrm{L} / \mathrm{B}$ & \multicolumn{3}{|c|}{5.116} \\
\hline $\mathrm{B} / \mathrm{d}$ & \multicolumn{3}{|c|}{3.473} \\
\hline $\mathrm{CB}_{\mathrm{B}}$ & 0.5668 & 0.5696 & 0.5752 \\
\hline $\mathrm{CBF}$ & 0.5954 & 0.6014 & 0.6136 \\
\hline $\mathrm{CBA}$ & \multicolumn{3}{|c|}{0.5423} \\
\hline $\mathrm{CM}_{\mathrm{M}}$ & \multicolumn{3}{|c|}{0.984} \\
\hline$f\left({ }_{0}^{\circ} A \bowtie\right)$ & 0.0 & 7.50 & 13.40 \\
\hline $1\left({ }_{0}^{\circ} L P P\right)$ & 0.0 & 4.30 & 4.30 \\
\hline
\end{tabular}

perimentally analyse the resistance components. The body plan of the forebody and the configuration of the flat bulb (B1) and the horizontal cylinder bulb (B2) are present in Fig. 8. Further details of the configuration, wave pattern pictures with and without these bulbs and results of resistance test are seen in Refs. 4) and 5).

Analysed wave profiles are compared in Figs. 9 and 10 at $F_{n}=0.22$ and 0.26 , respectively. The wave profiles in solid line is reproduced on the test line of $y / L=0.1057$ from the amplitude function of the wave record of the same $y$-coordinate, and those in dotted line is reproduced on the same test line from the amplitude function of the wave record at $y / L=0.2957$ where the nonlinearity of the wave is scarcely observed. At $F_{n}=0.22$ the waves are remarkably attenuated by both the flat bulb $\mathrm{B} 1$ and the horizontal cylinder bulb B2, and the difference between the solid and dotted lines, that corresponds to

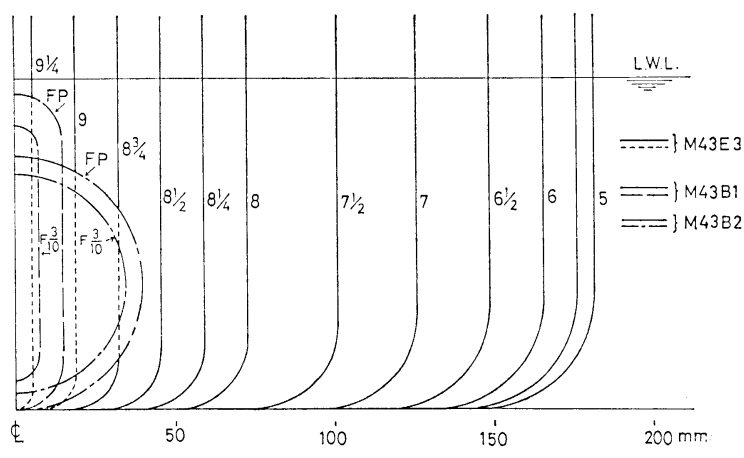

Fig. 8 Body plan of M43E3 bulb series

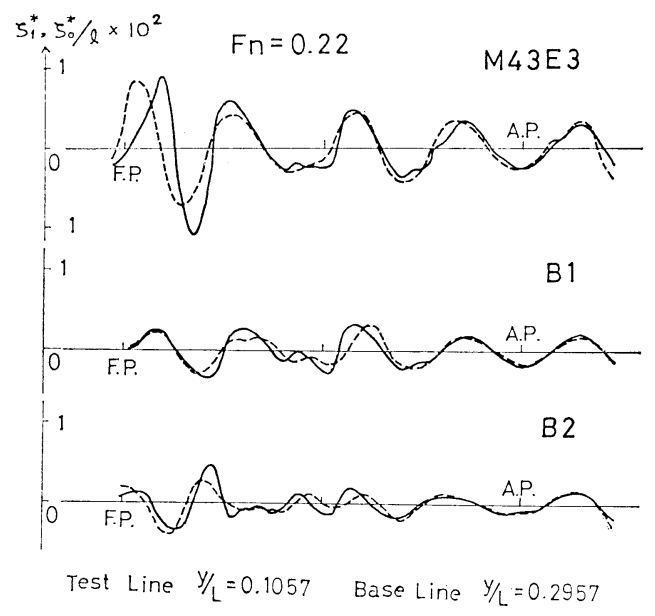

Fig. 9 Analysed longitudinal wave profiles, $\zeta_{1}^{*}$ and $\zeta_{0}^{*}$, of M43E 3 bulb. series at $F_{n}=0.22$ 


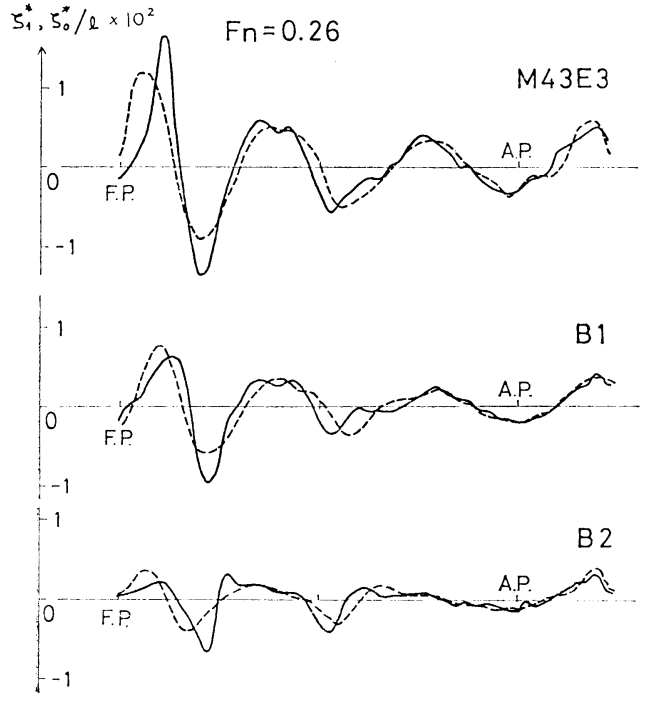

Fig. 10 Same as Fig. $9\left(F_{n}=0.26\right)$

$1 h$, is decreased by attaching bulbs. This indicates that the bulbs for M43E3 reduce nonlinear waves in the near-field and simultaneously reduce linear waves which is despersively spread to the far-field. At $F_{n}=0.26$ the differences between the two wave profiles are still remarkable for the models with bulbs, but it is small in comparison to M43E3 without a bulb.

The horizontal cylinder bulb is more effective for M43E3 than the flat bulb. However, the superiority of the former bulb almost disappears at $F_{n}=0.22$.

\section{Effect of frame-lines}

It has been pointed out through experimental investigations into the structure of FSSW that the flow with very large disturbance velocities is present in the thin layer near the free surface involving discontinuity of flow velocity and turbulence of the free surface. The experimental results on the effect of bulbs in the previous chapter indicate that the attenuation of disturbance velocities near the free surface results in reduction of FSSW. These implies that the modification of water-lines near the free surface influences the occurrence of FSSW and that the nonlinear component of wave resistance can be reduced by modifying the frame-lines of the forebody with the sectional area curve unchanged.

Following the first report, wave resistance is assumed to be decomposed into the linear component that corresponds to wave pattern resistance $\left(C_{w p}\right)$ and nonlinear component that is given by the difference of wave resistance and wave pattern resistance $\left(C_{w}-C_{w p}\right)$. The latter component has been often called resistance component due to FSSW by the authors. However, the detailed investigation into the structure of FSSW $^{3)}$ suggests that it is more proper to call it nonlinear component of wave resistance, because FSSWs can partly be a source of linear dispersive waves.

\subsection{Tested model and variation of frame-lines}

A hull form of a fine $\mathrm{RO} / \mathrm{RO}$ container carrier is chosen for the present purpose. The $2 \mathrm{~m}$ model of this hull form is called M51 and its particulars are shown in Table 2. The design Froude number is 0.267 .

The frame-lines of the forebody of this hull form is systematically changed keeping the sectional area curve unchanged as described below. The half-breadth of the load-water-line at longitudinal coordinate of $x$ is reduced by $\Delta b$ which is given by the following equation.

$$
\Delta b(x)=B \cdot|x| \cdot\{1-\cos (2 \pi x)\}
$$

$\Delta b, B$ and $x$ are nondimensionalized by the halflength of the ship (l), and the origin of the $x$ -

Table 2 Principal particulars of M51 and R014

\begin{tabular}{|l|l|l|}
\hline$(\mathrm{m})$ & $\mathrm{M} 51$ & $\mathrm{R} 014$ \\
\hline $\mathrm{Lpp}$ & 2.000 & 4.500 \\
\hline $\mathrm{B}$ & 0.333 & 0.750 \\
\hline $\mathrm{d}$ & 0.098 & 0.2205 \\
\hline $\mathrm{L} / \mathrm{B}$ & \multicolumn{2}{|c|}{6.0} \\
\hline $\mathrm{B} / \mathrm{d}$ & 3.4 \\
\hline $\mathrm{CB}$ & 0.5499 \\
\hline $\mathrm{CBF}$ & 0.5061 \\
\hline $\mathrm{CBA}$ & 0.5937 \\
\hline $\mathrm{CM}$ & 0.9697 \\
\hline
\end{tabular}

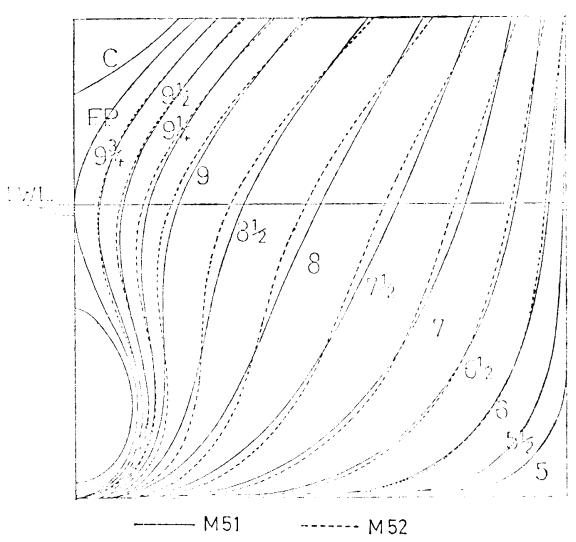

Fig. 11 Body plans of forebodies of M51 and M52 
coordinate is located at the midship. $x$ at FP is -1 and midship zero. The value $B$ is systematically varied. Being given the value of the reduction of beam-length at each $x$-coordinate on the load-water-line, the frame-lines are varied by adding the following value to the original offsets.

$$
1 b(x) \cdot \cos (\pi z / d)
$$

$z$ is the vertical coordinate and $z / d$ varies from zero to 1 , and therefore, the sectional area curve at each longitudinal location is kept unchanged. When $B$ is a positive value, a part of the volume of the hull near the free surface is transferred to the deeper region. The value of $B$ is varied from -0.01 to 0.015 .

\subsection{Method of estimating wave resistance}

The wave resistance of the modified hull forms is estimated under the two basic assumptions, i.e., that wave resistance and wave pattern resistance of the original hull form is given beforehand from towing test and wave analysis and that wave resistance is lecomposed into the linear component $C_{w p}$ and the nonlinear component $C_{w: s}\left(=C_{w-} C_{w p}\right)$.

The linear component is obtained by multiplying the calculated wave resistance $\left(C_{w l}\right)$ by $m$. $C_{w l}$ is calculated by one of the linear wave resistance calculation programs, i.e., $\mathrm{HSFW}^{6)}$, and $m$ is determined by dividing the $C_{w p}$ of the original hull analysed from wave analysis by the calculated $C_{w l}$ of the same hull form. It is a modification factor and is 0.679 for the present case at $F_{n}=0.267$.

The nonlinear component of wave resistance $C_{w s}$ is estimated by the following procedure which is based on the concept of equivalent shallow water depth. ${ }^{2)}$

1) The shock angle $(\beta)$ is calculated by the method of characteristics assuming that the hull form is represented by the load-water-line curve. The equivalent shallow water depth is so determined that the calculated $\beta$ of the original hull form accords with the measured, and it is assumed not to depend on the frame-line modification.

2) From the shock angle $\beta$ calculated above, energy deficit ratio $\Delta e$ is calculated by the nonlinear shallow water theory (for details see Chapter 5 of Ref. 2)). In this calculation it is postulated that a wedge model in shallow water that generates shock angle of $\beta$ stands for the hull form.

The relation of $C_{w s}, \Delta e$ and $\beta$ is shown in Fig. 12 for the four series models tested in the first report, and that of $\Delta e$ and $C_{w s}$ is shown in Fig. 13. De seems to have close connection with $C_{w s}$ that is derived as $C_{w}-C_{w p}$, and they are approximately in linear relation. The experimentally obtained $C_{w s}$ and calculated $\Delta e$ of the original hull form M51 is plotted by a dark circle in F: $\mathrm{g}$.

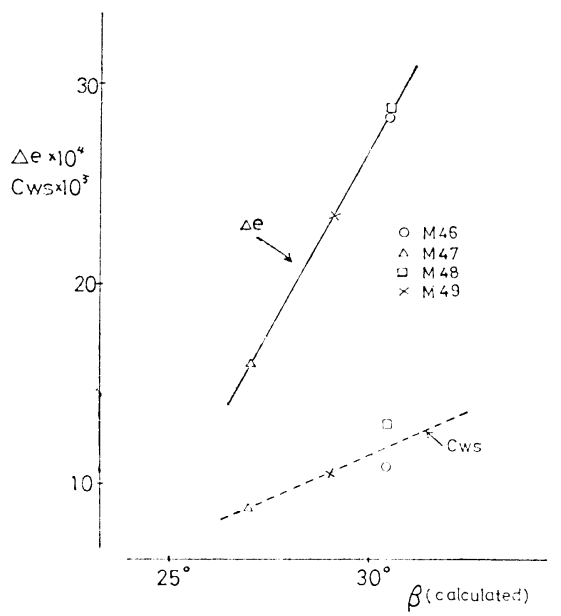

Fig. 12 Relation between $\Delta e, C_{w s}$ and shock angle

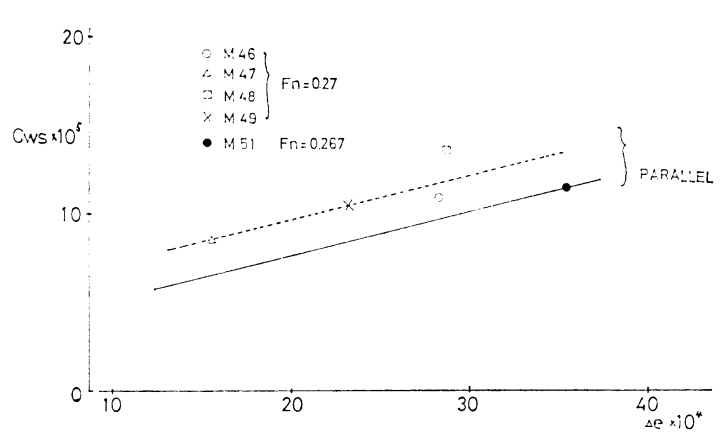

Fig. 13 Relation between $\Delta e$ and $C_{w s}$

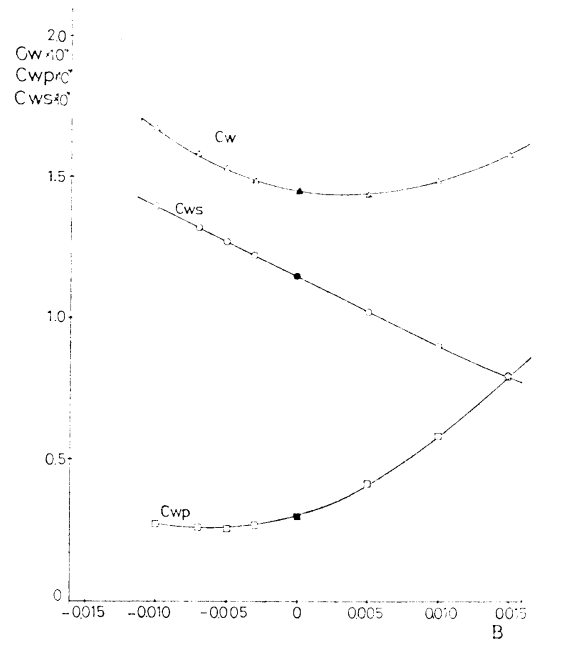

Fig. 14 Estimated $C_{w s}, C_{w l}$ and $C_{w}$

13, and $C_{w s}$ of the modified hull form is assumed to be estimated by the solid line in Fig. 13 from the calculated $\Delta e$. 
The estimated $C_{w}$ of modified models is derived from the summation of $C_{w p}\left(m \times C_{w l}\right)$ and $C_{w s}$ from Fig. 13. The estimated $C_{w}, C_{w s}$ and $C_{w p}$ are shown in Fig. 14. Increasing $B$, namely decreasing the water-plane area, leads to increase of $C_{w p}$ and decrease of $C_{w s}$. Fine entrance reduces FSSW, while it intensifies linear waves in this case. $C_{w}$ of the original hull form is close to the minimum value.

The advantage of the present method is that it necessitates experiments on only one original full form, while the method in the first report requires at least three ship models to be tested beforehand.

\subsection{Experimental results and discussion}

The $B$ value of 0.005 is chosen for the modification of the original model M51. The modified model M52 is as shown in Fig. 11 and Table 2. M52 is manufactured from paraffin wax to be served for some experiments to know the validity of the present method of estimation which can be applicable to hull form improvement. $C_{w p}$ of M52 is expected to be greater than M51 and on the contrary $C_{w s}$ smaller, and finally the total wave resistance will be slightly reduced by the modification of the frame-lines.

The results of towing test and wave analysis of M52 is compared to those of M51 in Fig. 15. As expected, $C_{w}$ is reduced by the modification,

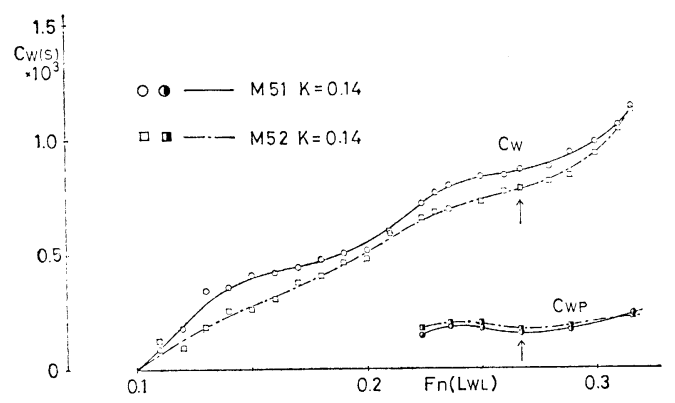

Fig. $15 C_{w}$ curves of M51 and M52

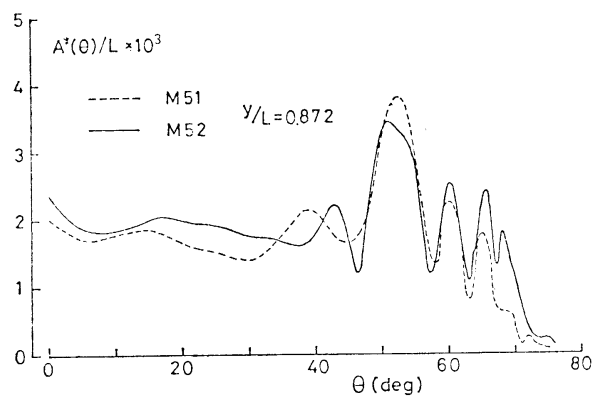

Fig. 16 Weighted amplitude function of M51 and M52 at $F_{n}=0.267$

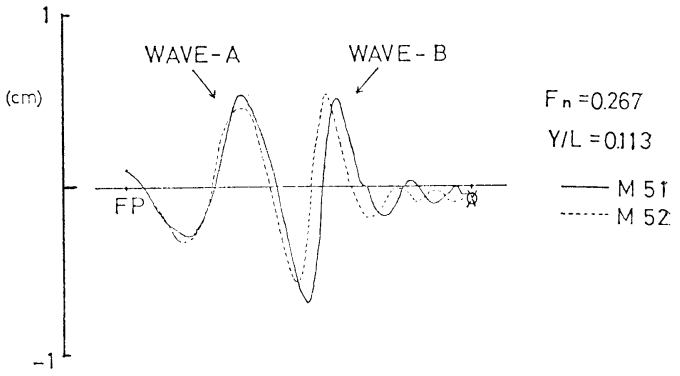

Fig. 17 Extracted longitudinal wave profiles of M51 and M52 at $F_{n}=$ 0.267

whereas $C_{w p}$ is increased. However, the degree of increase of $C_{w p}$ is smaller than expected, and consequently the reduction of $C_{w}$ turned out to be a little greater. The increase of wave pattern resistance is revealed in the weighted wave amplitude function shown in Fig. 16.

The decrease of $C_{w s}$ due to the modification of frame-lines can be exemplified by the analysis. of longitudinal wave profiles on the longitudinal lines near and far from the hull in the manner proposed in Chapter 3 of the previous paper. The test-line and the base-line chosen for the present models are at $y / L=0.113$ and 0.262 , respectively. The extracted wave profiles $h(x)$ for M51 and M52 are compared in Fig. 17. Since the extracted wave profile corresponds to the magnitude of the noninear component of wave resistance, Fig. 17 indicates that the frame-line modification of M51 into M52 reduces $C_{w s}$ as expected. This analysis of wave profiles demonstrates the qualitative validity of the present method of estimating the nonlinear component of wave resistance.

Wave height contours of M51 are drawn by the usual method and compared to those of R014 which is a geosim model of M51 whose length is $4.5 \mathrm{~m}$ and tested at the Shipbuilding Research Center of Japan. The two contours in Fig. 18, in general, accord well between each other and the law of similarity is almost exemplified, although the crest and trough of the waves generated by the smaller model is attenuated to a certain degree. The Froude's law of similarity is approximately satisfied by the wave formation in the near-field, involving some scale effect.

\section{Conclusion}

Principal conclusions are as follows.

1) The effect of bow-bulbs properly designed is apparently equivalent to sharpening the entrance.

2) The effect of bow-bulbs consists in reducing 


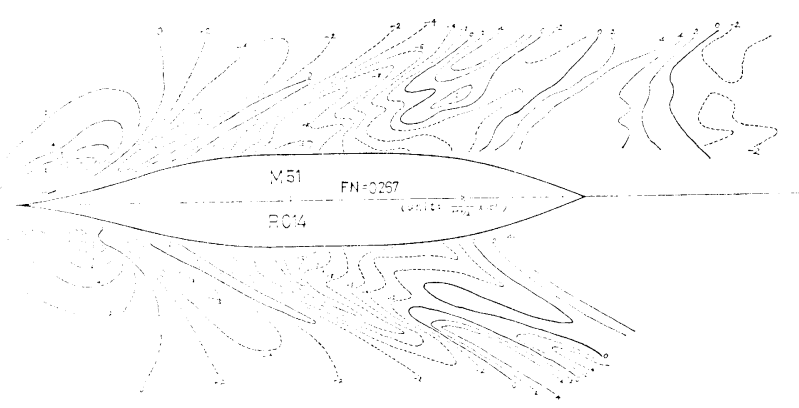

Fig. 18 Wave height contours of geosim models, M51 and R014

the otherwise very great disturbance velocity caused by bows in the flow field near the free surface.

3) The resistance due to free surface shock waves can be reduced by modifying the framelines without changing the sectional area curve.

4) The hybrid method of estimating wave resistance, which is based on both the linear wave making theory and the nonlinear shallow water wave theory, is useful for the improvement of frame-line configurations.

The methods proposed in the previous and present papers are based on some bold postulations. However, they can be available for practical design procedures as demonstrated. The method of estimating the nonlinear component of wave resistance is mostly connected with the configuration of hull forms near the bow. The hull forms behind bows should be taken into account for the further improvement of the proposed methods in near future, because they pressumably influence the generation of free surface shock waves.

\section{References}

1) Nito, M. et al.: Free Surface Shock Waves and Methods for Hull Form Improvement (First Report), J. Soc. Naval Arch. Japan, Vol. 150, (1981).

2) Takahashi, M. et al.: Characteristics of Free Surface Shock Waves around Wedge Models, J. Soc. Naval Arch. Japan, Vol. 148 (1980)

3) Miyata, H. et al.: Characteristics of Free Surface Shock Waves around Wedge Models (Second Report), J. Soc. Naval Arch. Japan, Vol. 151 (1982).

4) Kawamura, $N$. et al.: Experimental Investigation on the Resistance Component Due to Free Surface Shock Waves on Series Ship Models, J. Kansai Soc. Naval Arch., Japan, Vol. 179 (1980).

5) Inui, $T$. et al.: From Bulbous Bow to Free-Surface Shock Wave-Trends of 20 Years' Research on Ship Waves at the Tokyo University Tank, J. Ship Research, Vol. 25, No. 3 (1981).

6) Miyata, H. et al.: On the Optimization of the Aft-Part of Fine Hull Forms (First Report), J. Kansai Soc. Naval Arch., Japan, Vol. 179 (1980). 